\title{
Wie Nervenzellen miteinander reden - molekulare Mechanismen der Neurotransmitter-Freisetzung
}

Nervenzellen oder Neurone sind Grundbestandteile unseres Nervensystems. Sie sind spezialisiert auf Empfang, Verrechnung, und Weiterleitung von elektrischen Signalen. Die Signalübertragung von einem Neuron auf ein anderes Neuron oder eine nicht-neuronale Zielzelle, beispielsweise eine Muskelzelle, erfolgt an spezialisierten Kontaktstellen, den Synapsen. Hier werden sowohl an der Senderzelle wie der Empfängerzelle Spezialisierungen ausgebildet, die man als prä- und postsynaptisches Kompartiment bezeichnet. In Synapsen werden die elektrischen Signale in chemische Signale umcodiert. Dabei werden in den präsynaptischen Nervenenden der Senderzelle kleinmolekulare Substanzen, die Neurotransmitter, freigesetzt, die von Rezeptoren in der Membran der Empfängerzelle ausgelesen und

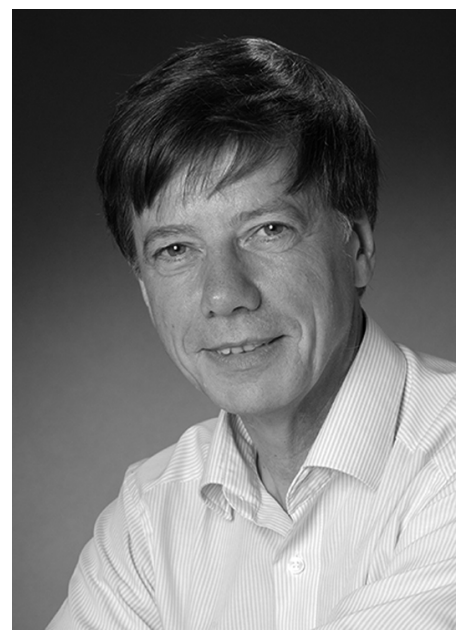

Reinhard Jahn, Honorarprofessor für Biologie, O. Mitglied der Akademie seit 2015 wieder in elektrische Signale umgewandelt werden. Allerdings werden die Signale nur ausnahmsweise unverändert übertragen, so z. B. an den die Muskelkontraktion steuernden neuromuskulären Endplatten. Meistens kommt es an Synapsen zu einer Verstärkung oder Abschwächung des Signals, z.B. dadurch bedingt, dass sich eine Synapse an die vergangene Aktivität „erinnert“ und ihren Durchsatz dynamisch anpasst, was auch als synaptische Plastizität bezeichnet wird.

Die Freisetzung von Neurotransmittern ist ein komplexer Vorgang, der in den letzten Jahrzehnten in vielen Details aufgeklärt worden ist (1, 2, s. Abb. 1). Neurotransmitter werden im Zytoplasma der Nervenenden synthetisiert und dann in kleine membranumhüllte Bläschen, die synaptischen Vesikel, verpackt. Eine einzelne Synapse im Gehirn enthält üblicherweise einige Hundert dieser Vesikel, doch gibt es auch Spezialisten unter den Synapsen, in denen die Zahl der Vesikel viele Hunderttausend betragen kann (z.B. in der oben erwähnten neuromuskulären Endplatte). Wenn ein elektrisches Signal in einem Nervenende eintrifft, ändert sich die elektrische Spannung über der Membran von ca. $-70 \mathrm{mV}$ auf kurzzeitig +20-30 mV. In der Plasmamembran der Nervenenden befinden sich Ionenkanäle, die auf diese Spannungsänderung reagieren und vom „off“ in den 
„on“ Zustand übergehen. Dadurch strömen Calcium-Ionen vom Extrazellulärraum in das Innere der Nervenenden. Dort lösen die Calcium-Ionen eine Verschmelzung der Vesikel- mit der präsynaptischen Plasmamembran aus (Exocytose), wodurch der in den synaptischen Vesikeln gespeicherte Neurotransmitter freigesetzt wird. Die Transmittermoleküle diffundieren über den synaptischen Spalt, der die Sender- von der Empfängerzelle trennt. Dort treffen sie wiederum auf schaltbare Ionenkanäle, die durch die Bindung der Transmittermoleküle kurzzeitig eingeschaltet werden. Dadurch können Ionen über die Membran wandern, was eine Änderung der Membranspannung in der Empfängerzelle bewirkt, d.h. aus einem chemischen ist wieder ein elektrisches Signal entstanden.

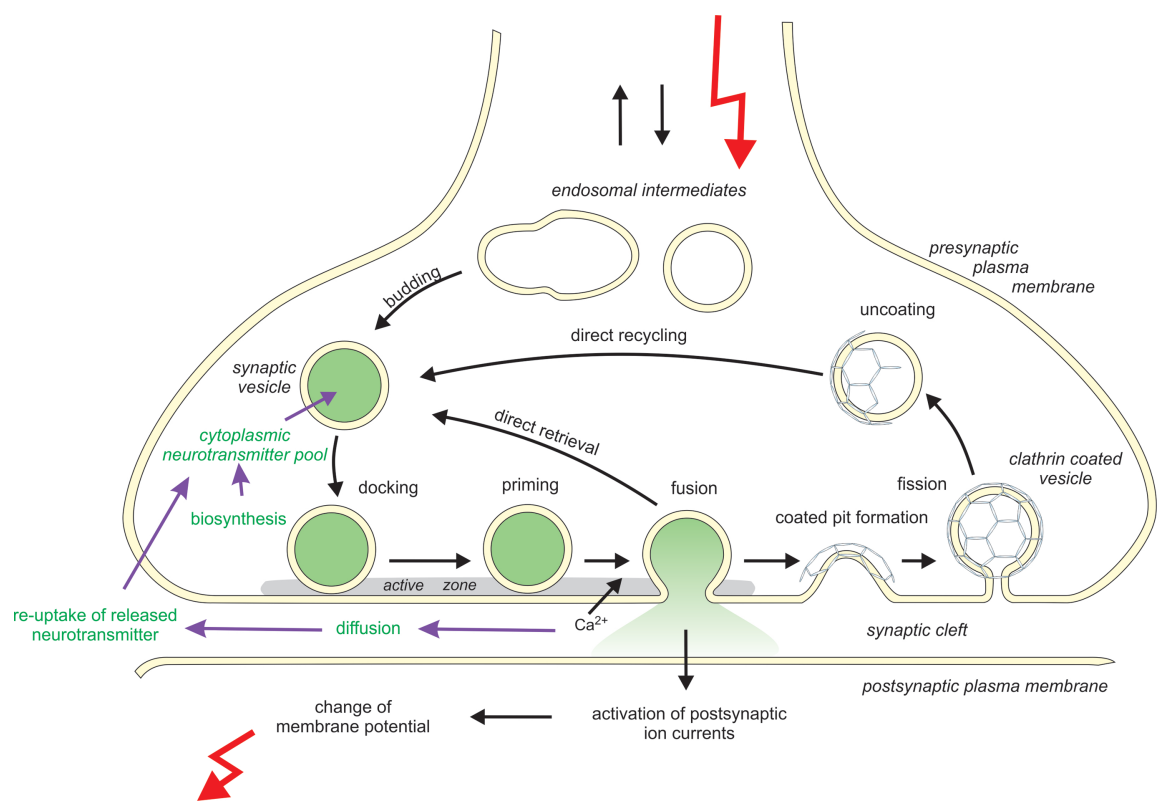

Abb. 1: Mechanismus der Signalübertragung an einer chemischen Synapse.

Auf der präsynaptischen Seite wird die Vesikelmembran durch Endocytose wieder in die Zelle aufgenommen und vermutlich über einen oder mehrere Zwischenschritte wieder zur Bildung von synaptischen Vesikeln benutzt, wodurch ein geschlossener Membrankreislauf gebildet wird (Abb. 1).

Die molekularen Grundlagen der Calcium-vermittelten Exocytose sind in den letzten zwei Jahrzehnten weitgehend aufgeklärt worden. Die Membranfusion selbst wird durch eine Gruppe von Proteinen bewerkstelligt, die als SNARE-Proteine bezeichnet werden. Diese Proteine operieren als Nanomaschinen, und zwar auf folgende Weise (Abb. 2). 


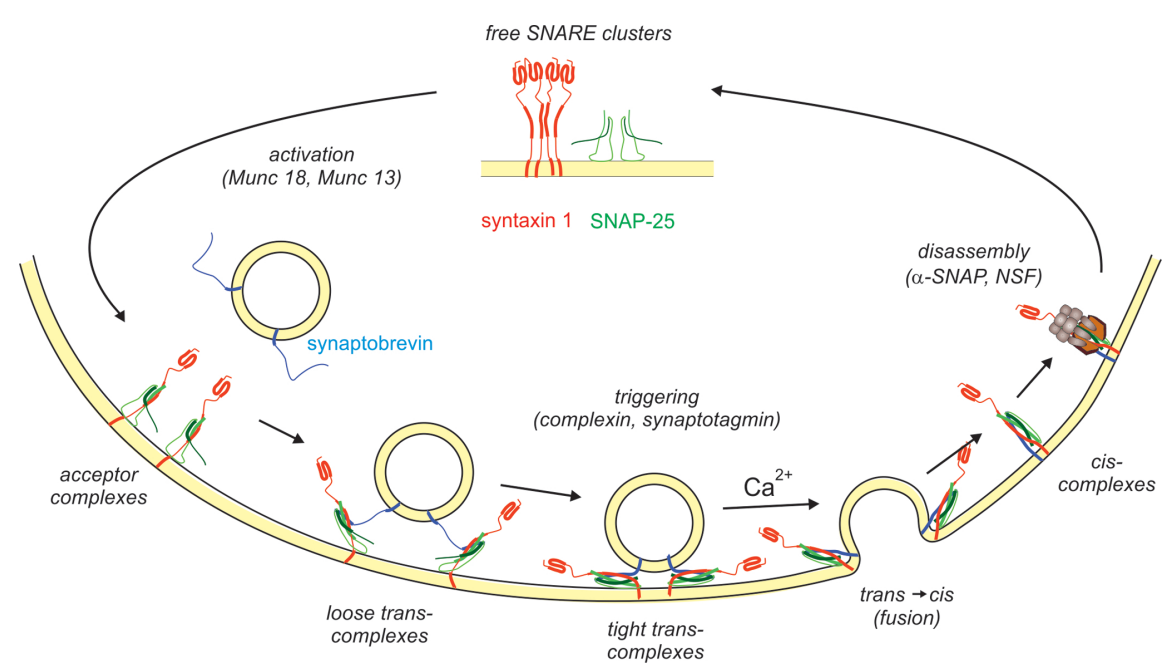

Abb. 2: Molekularer Mechanismus der SNARE-vermittelten Membranfusion.

In der Membran synaptischer Vesikel sowie in der Plasmamembran befinden sich komplementäre SNARE-Proteine, die, wenn sie mit ihren Enden miteinander in Kontakt kommen, sich spontan miteinander zu $\alpha$-helikalen Bündeln verdrillen, wodurch die Membranen fest aneinander gezogen werden. Dabei wird die elektrostatische Abstoßung zwischen den Membranen überwunden, und es kommt zur Ausbildung von hydrophoben Kontakten, die über noch nicht in allen Einzelheiten verstandene Zwischenschritte zur vollständigen Verschmelzung der Membranen führen. Nach der Fusion ist die in den SNARE-Molekülen gespeicherte konformationelle Energie verbraucht. Sie müssen mit Hilfe eines Spezialproteins, der AAA+-ATPase NSF, wieder ,aufgedröselt“ und damit für eine erneute Verwendung reaktiviert werden (3, Abb. 2).

Die Entdeckung der SNARE Proteine und die Erkenntnis, dass diese Proteine die Katalysatoren der Membranfusion sind, erfolgte zu Beginn der 90er Jahre und war für alle Beteiligten sehr aufregend. Individuelle SNARE Proteine wurden z.T. unabhängig in mehreren Arbeitsgruppen entdeckt, aber ihre Bedeutung blieb unklar. Der erste Hinweis auf eine Funktion in der Exocytose kam durch den überraschenden Befund, dass die SNARE Proteine durch eine Gruppe bakterieller Toxine angegriffen werden. Dabei handelt es sich um die Botulinus und Tetanus Neurotoxine, die bei einer Infektion in den Blutstrom gelangen können und die für ihre Hemmung der neuronalen Signalübertragung berüchtigt sind. Unabhängig davon stellte sich heraus, dass diese Proteine mit der oben erwähnten ATPase NSF interagieren, die zuvor als ein für die Fusion wichtiger Faktor identifiziert worden war. Ebenfalls zur selben Zeit wurde erkannt, dass die Proteine mit 
Proteinen in der Hefe verwandt sind, denen ebenfalls eine Rolle im intrazellulären Membrantransport zugeschrieben wird (4).

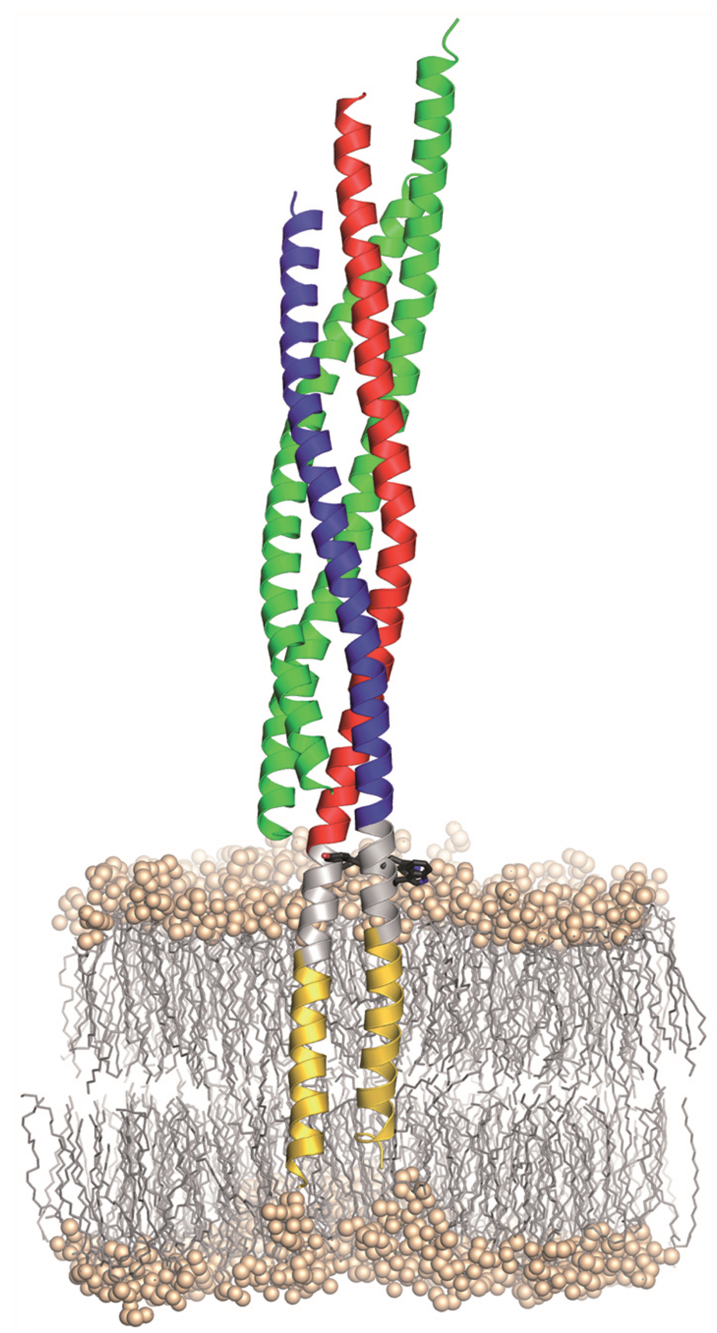

Abb. 3: Kristallstruktur eines neuronalen SNARE-Komplexes mit Membrananker (aus 6).

Die Struktur des SNARE-Komplexes, die in Zusammenarbeit mit dem Labor von Axel Brunger (Yale University, jetzt Stanford University), einige Jahre später aufgeklärt werden konnte, bildet bis heute eine wichtige Grundlage für zahlreiche detaillierte Untersuchungen des SNARE-vermittelten Fusionsmechanismus in vielen Arbeitsgruppen (5). Vor einigen Jahren konnte A. Stein, in Zusammenarbeit 
mit der Gruppe von Markus Wahl, auch die Struktur der SNARE-Membrananker sowie der funktionell wichtigen Verbindung zwischen SNARE-Domäne und dem Membrananker aufklären (Abb. 3, 6).

Ebenfalls Anfang der neunziger Jahre hat meine Gruppe, in enger Zusammenarbeit mit dem Labor von Thomas C. Südhof, das Protein Synaptotagmin identifiziert und nachgewiesen, dass es als Calcium-Sensor in der Exocytose funktioniert (7). Seither sind eine Reihe weiterer Proteine entdeckt worden, die alle dazu dienen, die Funktion der SNARE-Proteine als Katalysatoren der Membranverschmelzung zu regulieren.

In meiner eigenen Gruppe verwenden wir rekombinant hergestellte Proteine und künstliche Membranen, um den Mechanismus der Membranfusion besser zu verstehen. Hierbei werden verschiedene Typen (incl. genetisch veränderter Varianten) von SNARE Proteinen unter genau kontrollierten Bedingungen in künstliche Vesikel (sog. Liposomen) eingebaut und die Bindung sowie Fusion der Vesikel untereinander mit verschiedenen Verfahren gemessen. Ziel dieser Arbeiten, die teilweise in Zusammenarbeit mit weiteren Gruppen aus Göttingen durchgeführt werden, ist es, die einzelnen Schritte und Zwischenzustände, die bei der Verschmelzung der Membranen durchlaufen werden, genau zu verstehen (siehe z. B. 8). Eins der Probleme besteht darin, dass die Vorgänge sehr schnell sind, was kinetisch kontrollierte Messungen erfordert. Zudem sind diese Vesikel alle kleiner als die Auflösungsgrenze der besten Mikroskope, so dass man auf teilweise aufwändige und indirekte Nachweisverfahren angewiesen ist, aus denen Rückschlüsse auf die Zwischenstufen nicht immer eindeutig zu erzielen sind. Um mögliche Artefakte durch die Verwendung solch künstlicher Systeme ausschließen zu können, verwenden wir auch gereinigte biologische Membranen, die mit unseren künstlichen Vesikeln in spezifischer Weise fusionieren. Umgekehrt haben wir künstliche Vesikel in intakte Zellen eingeführt, um herauszufinden, welche Faktoren für eine Erkennung bzw. Fusion mit dem korrekten Zielkompartiment erforderlich sind.

Neben den Arbeiten am Fusionsmechanismus interessieren wir uns für die Struktur und Funktion synaptischer Vesikel. So wurde von uns vor einigen Jahren ein quantitatives strukturelles Modell eines prototypischen synaptischen Vesikels erarbeitet, das seither als Modell für ein typisches Transportvesikel stark beachtet worden ist (9, Abb. 4).

Zudem erforschen wir seit vielen Jahren die Mechanismen, die der Beladung der Vesikel mit Neurotransmittern zugrunde liegen. Diese Transportvorgänge werden durch spezifische Transportproteine bewerkstelligt, welche einen elektrochemischen Protonengradienten als Energiequelle benutzen, der von einer membranständigen Protonenpumpe erzeugt wird. Hierbei haben wir uns insbesondere auf die Aufnahme des jeweils wichtigsten erregenden (Glutamat) und hemmenden 
(GABA) Neurotransmitters konzentriert. Auch hierbei verwenden wir gereinigte Proteine sowie künstliche Membranen, um die Transportmechanismen besser zu verstehen.

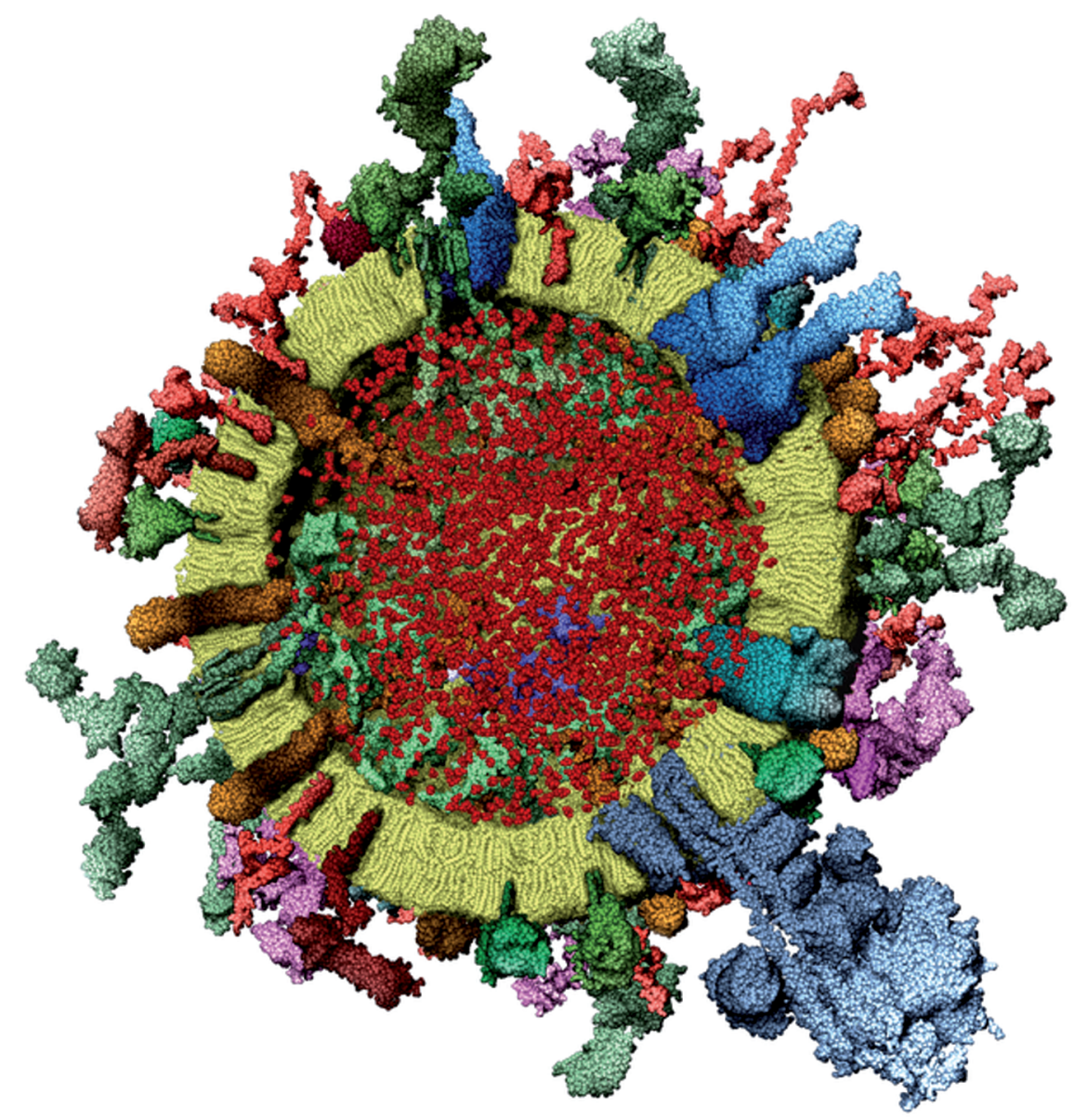

Abb. 4: Molekularmodell eines synaptischen Vesikels. Die Neurotransmitter-Moleküle sind als rote Punkte im Inneren des aufgeschnittenen Vesikels dargestellt. Die Membranlipide sind gelb, die Membranproteine in verschiedenen Farben dargestellt. Die Abbildung zeigt ein Strukturmodell der wichtigsten Proteine und Lipide im richtigen Mengen- und Größenverhältnis.

Eine Besonderheit der Neurotransmitter-Aufnahme besteht darin, dass sie während des gesamten Transportvorgangs mit gleichbleibender Effizienz ablaufen muss, obwohl sich dabei die ionische und osmotische Zusammensetzung des Vesikel-Inhaltes gewaltig verändert. Wir haben herausgefunden, dass diese Flexi- 
bilität durch fakultativ besetzte Ionenbindungsstellen und Gegen-Transportmechanismen erreicht wird, wodurch die Transportproteine den Transportmechanismus während des „Auftankens“ an die veränderten Bedingungen anpassen können. Zudem ist es uns gelungen, solche Prozesse an einzelnen Vesikeln mit Hilfe von neuartigen fluoreszent-mikroskopischen Verfahren zu messen, was eine wesentlich verbesserte Zeitauflösung sowie neue mechanistische Einsichten ermöglicht hat (10-12).

\section{Literatur}

1. Südhof, T.C. (2013) Neurotransmitter release: the last milliscecond in the life of a synaptic vesicle. Neuron $80,675-690$

2. Rizo, J., Xu, J. (2015) The synaptic vesicle release machinery. Annu. Rev. Biophys. 44, 339-367

3. Jahn, R., Scheller, R.H. (2006) SNAREs - engines for membrane fusion. Nature Reviews Mol. Cell Biol. 7, 631-643

4. Jahn, R. (2008) Some classic papers in the field of membrane fusion - a personal view. Nature Struct. Mol. Biol. 15, 655-657

5. Sutton, B., Fasshauer, D., Jahn, R., Brünger, A.T. (1998) Crystal structure of a SNARE complex involved in synaptic exocytosis at 2.4 A resolution. Nature 395, 347-353

6. Stein, A., Weber, G., Wahl, M.C., Jahn, R.(2009) Helical extension of the neuronal SNARE complex into the membrane. Nature 460, 525-528

7. Brose, N., Petrenko, A.G., Südhof, T.C., Jahn, R. (1992) Synaptotagmin: a Ca2+ sensor on the synaptic vesicle surface. Science 256, 1021-1025

8. Hernandez, J.M., Stein, A., Behrmann, E., Riedel, D., Cypionka, A., Farsi, Z., Walla, P.J., Raunser, S., Jahn, R. (2012) Membrane fusion intermediates via directional and full assembly of the SNARE complex. Science, 336, 1581-1584

9. Takamori, S., Holt, M., Stenius, K., Lemke, E.A., Grønborg, M., Riedel, D., Urlaub, H., Schenck, S., Brügger, B., Ringler, P., Müller, S.A., Rammner, B., Gräter, F., Hub, J.S., De Groot, B.L., Mieskes, G., Moriyama, Y., Klingauf, J., Grubmüller, H., Heuser, J., Wieland, F., Jahn, R.(2006) Molecular anatomy of a trafficking organelle. Cell 127, 831-846

10. Takamori, S., Rhee, J.-S., Rosenmund, C., Jahn, R. (2000) Identification of a vesicular glutamate transporter that defines a glutamatergic phenotype in neurons. Nature 407, $189-194$

11. Preobraschenski, J., Zander, J.-F., Suzuki, T., Ahnert-Hilger, G., Jahn, R.:(2014) Vesicular glutamate transporters (VGLUTs) use flexible anion and cation binding sites for efficient accumulation of neurotransmitter. Neuron 84, 1287-1301

12. Farsi, Z., Preobraschenski, J., van den Bogaart, G., Riedel, D., Jahn, R., Woehler, A. (2016) Single-vesicle imaging reveals different transport mechanisms between. glutamatergic and GABAergic vesicles. Science, in press. 